\title{
$\Leftrightarrow$ SkınMedica
}

\section{BACKGROUND}

Similar to facial skin aging, visible features of aged lips can be attributed to
degradation of structural components of the skin including collagen and degradation of structural components of the skin including collagen and
elastin fibers. Key features of lip aging include loss of volume, color, and definition as well as increases in lines/wrinkles and uneven skin texture. As a result these factors result in the loss of contrast between lips and
surrounding skin and is associated with an older, aged appearance. OBJECTIVE To assess the efficacy and tolerability of a novel, topical two-step lip
treatment that contains hyaluronic acid $\left(H A^{5}\right.$ LS).

STUDY DESIGN

Open-label, single-center clinical usage study.

- Four week study duration with visits at baseline, week 2 and week 4 . Subject Inclusion Criteria

Female subjects aged 22-40 years with Fitzpatrick Skin Types (FST) I to V who have self-perceived and clinically determined average-size lips. Must be a non-smoker and have not smoked within the last 5 years. Must have mild to moderate dryness on the lips at baseline with a score between 3 and 6 on modified Griffiths' scale. Must have mild to severe lip condition (lip texture/visual roughness and
lines/wrinkles) with a score of 3 or higher on the modified Grifiths' scale.

Treatment

Subjects were instructed to apply HA $\mathrm{H}^{5} \mathrm{LS}$ (Step 1: Lip Treatment, Step 2: Lip Plumper) at a minimum of three times a day to ensure coverage 8 hours a prior to each follow-up visit, but not within one hour of the visit.

Clinical Assessments

To capture the immediate effects of applying $\mathrm{HA}^{5} \mathrm{LS}$, clinical grading and on the lips. Long-term efficacy was assessed at week 2 and week 4 . The following efficacy parameters were assessed on the lips using a 0-9 scal where $0=$ none (best possible condition), $1-3=$ mild, $4-6=$ moderate, and
$7-9=$ severe (worst possible condition), with half points allowed as necessary to

- Lip Lines/Wrinkles Lip Color/Rosiness

- Overall Lip Definition/Lip Contour - Lip Plumpness

The efficacy parameters of cupping and scaling were assessed on a 0-6 scale, - Cupping: 0 =full, plump, $1=$ drawing at lip margin, $2=$ isolated area cupping at margin, $3=$ few areas cupping along lip margin, $4=$ cupping all along lip margin, $5=$ cupping more than lip margin, and $6=$ entire surface cuppin $4=$ generalized lifting $4=$ generalized lifting scale-mild,
$6=$ generalized lifting scale-severe

Tolerability assessments for erythema, edema, burning, stinging, itching and tightness were condus
$3=$ severe) at all visits.

Subject Self-Assessment Questionnaires

Subjects completed a self-assessment questionnaire at all visits.

Instrumentation

(Canfield Imagingographs were taken using the VISIA-CR Imaging week 2 and week 4 (pre- and post-application). A Alip wrinkle image analysis was conducted with a software developed by Stephens \& Associates using digital caliper was used to mesura Cybernetics, Inc., Rockville, Maryland). A digital caliper was used to measure lower lip plumpness, and Corneometer
CM 825 (Courage + Khazaka, Germany) was used to measure skin hydration.

\section{RESULTS}

Thirty-one female subjects, aged 22-40 years with FST II-V who identified as Caucasian, African American, Native (thalian or other Pacific Islander and Asian ethnicities, completed the study.

Subjects presented with mean scores of 3.11 for lip scaling (dryness) and 4.14 for overall lip condition. Five subjects voluntarily withdrew from the study (not due to adverse events).

- Immediate effects: Within 15 minutes of application, statistically significant improvements in mean scores compared to baseline were observed for all parameters (all $p<0.001$; Wilcoxon signed-rank test; $n=36$ ). - Long-term effects: Continuing significant improvements at weeks 2 and 4 compared to baseline for all
parameters, except for Cupping at Week 2 (all p $\leq 0.026$; Wilcoxon signed-rank test; Week 2: $n=32$, Week 4: $n=31$ ). - $\mathrm{HA}^{5} \mathrm{LS}$ was well-tolerated with no increase in mean tolerability scores at each evaluation time. By Week 4 , d during the study.

- The lip wrinkle image analysis supported the long-term improvements observed by the investigator, with significant improvements at week 4 post-application in all parameters (all $p \leq 0.001$; paired $t$-test; $n=31$ ). - Statistically significant improvements in mean scores for corneometer and digital caliper measurements at weeks
2 and 4 indicate an increase in intrinsic hydration and lip thickness, respectively, with continued use. - HA $A^{5} L S$ was highly rated by subjects with a statistically significant proportion of favorable responses for instant and erm self-perceived efficacy and product texture and attributes. At week 4 , a significant proportion of subjects felt that $\mathrm{HA}^{5} \mathrm{LS}$ made their lips look and feel more full $(90 \%)$

Figure 1: Instant Effects: Investigator Assessments (with product on the lips)

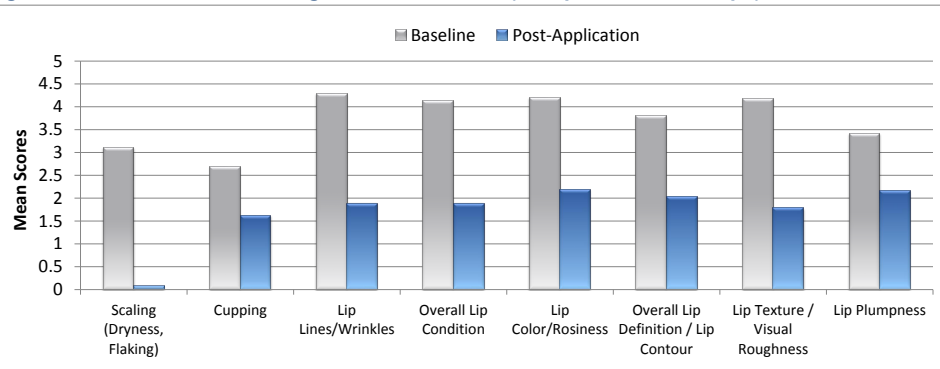

Figure 2: Instant Effects: Baseline vs. Baseline Post-Application (within 15 minutes of application)

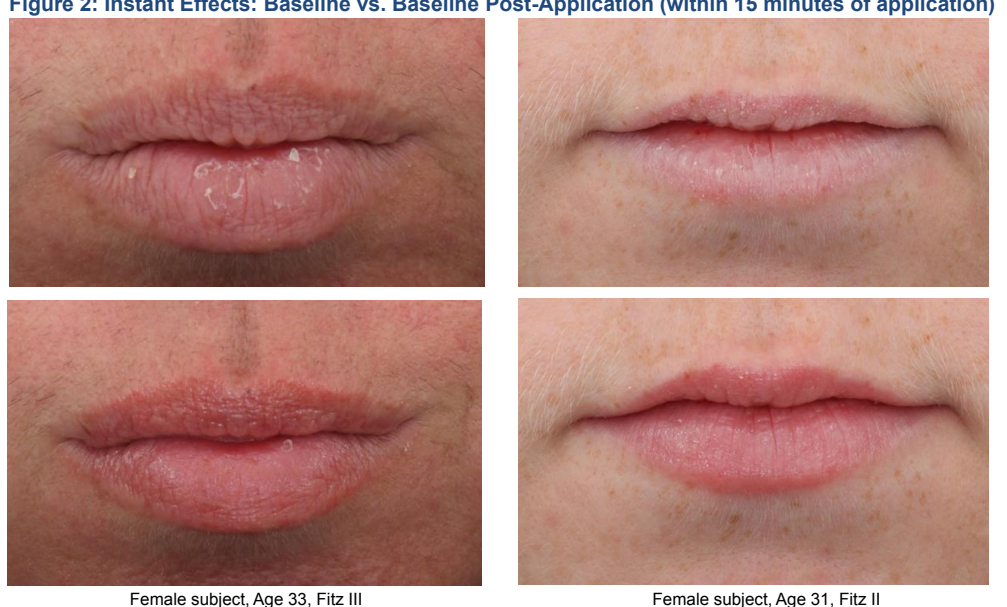

Figure 3: Long-Term Effects: Investigator Assessments (no product on the lips)

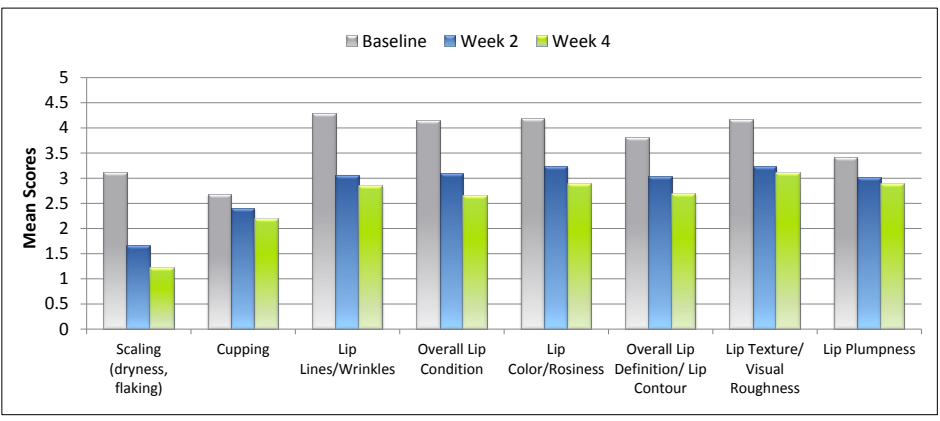

Figure 4: Long-Term Effects (no product on the lips)

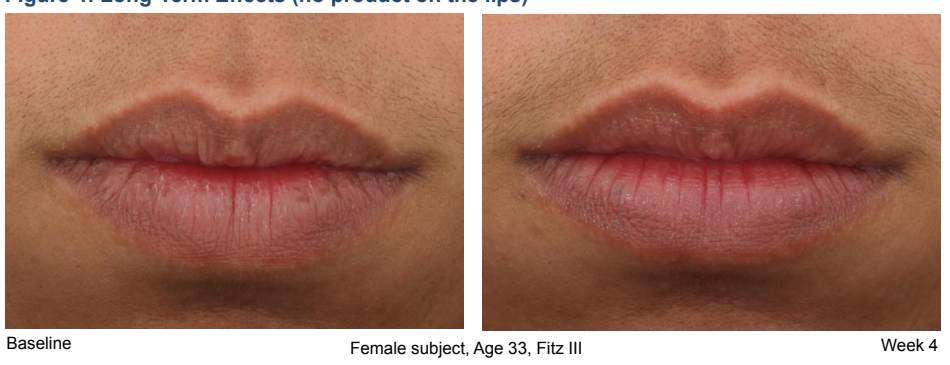

Figure 5: Lip Wrinkle Image Analysis on VISIA-CR Standard 2 Photographs

\begin{tabular}{|c|c|c|c|c|c|}
\hline & Total Count & $\begin{array}{l}\text { Total Length } \\
(\mathrm{mm})\end{array}$ & $\begin{array}{l}\text { Total Width } \\
(\mathrm{mm})\end{array}$ & $\begin{array}{l}\text { Total Area } \\
\left(\mathrm{mm}^{2}\right)\end{array}$ & $\begin{array}{l}\text { Total Depth } \\
\text { (arbitrary units) }\end{array}$ \\
\hline Baseline & 39.89 & 63.59 & 26.50 & 36.63 & 298.53 \\
\hline Baseline Post-Application & $27.80^{*}$ & $41.24 *$ & $17.25 *$ & $22.02^{*}$ & $174.34^{*}$ \\
\hline Week 4 Post-Application & $22.81^{*}$ & $33.86^{*}$ & $13.92^{*}$ & $17.94^{*}$ & $145.48^{*}$ \\
\hline
\end{tabular}

Figure 6: Corneometer and Digital Lip Caliper Measurements

\begin{tabular}{lcc}
\hline & $\begin{array}{c}\text { Corneometer Measurements for } \\
\text { Hydration (mean scores) }\end{array}$ & $\begin{array}{c}\text { Digital Lip Caliper Measurements for } \\
\text { Thickness in }\end{array}$ \\
\hline mm (mean scores)
\end{tabular}

CONCLUSIONS

Results from this study
and long-term benefits.

$H A^{5}$ LS may provide an alternative option to patients seeking a non-invasive treatment to rejuvenate and

Hestick.

DISCLOSURES 